Chapter 1

\title{
Complexities, Catastrophes and Cities: Emergency Dynamics in Varying Scenarios and Urban Topologies
}

\author{
Giuseppe Narzisi, Venkatesh Mysore, Jeewoong Byeon \\ and Bud Mishra \\ Courant Institute of Mathematical Sciences, New York University \\ 715 Broadway \#1002, New York, NY, USA \\ \{narzisi,mysore,jw.byeon,mishra\}@nyu.edu \\ Project website: www.bioinformatics.nyu.edu/Projects/planc
}

\section{Introduction \& Background}

Complex Systems are often characterized by agents capable of interacting with each other dynamically, often in non-linear and non-intuitive ways. Trying to characterize their dynamics often results in partial differential equations that are difficult, if not impossible, to solve. A large city or a city-state is an example of such an evolving and self-organizing complex environment that efficiently adapts to different and numerous incremental changes to its social, cultural and technological infrastructure [2]. One powerful technique for analyzing such complex systems is Agent-Based Modeling (ABM) [11], which has seen an increasing number of applications in social science, economics and also biology. The agentbased paradigm facilitates easier transfer of domain specific knowledge into a model. ABM provides a natural way to describe systems in which the overall dynamics can be described as the result of the behavior of populations of autonomous components: agents, with a fixed set of rules based on local information and possible central control. As part of the NYU Center for Catastrophe Preparedness and Response $\left(\mathrm{CCPR}^{1}\right)$, we have been exploring how ABM can

\footnotetext{
${ }^{1}$ visit: www.nyu.edu/ccpr
} 
serve as a powerful simulation technique for analyzing large-scale urban disasters. The central problem in Disaster Management is that it is not immediately apparent whether the current urban emergency plans are robust against such sudden, rare and punctuated catastrophic events. An agent-based emergency response model can utilize the large amount of information about the possible rules of behavior for people, hospitals, on-site responders and ambulances, without depending on the scarce knowledge about the efficacy of those rules or the global dynamics.

We have been striving towards a methodical and algorithmic approach for both preparedness and response, by combining powerful ideas from modelchecking, simulation and multi-objective optimization, in order that a large urban structure can recover from the effects of a disastrous event quickly and efficiently. Recently, game theoretic paradigms have also influenced the analysis of complex systems. In our models, persons play "games" with each other for the medical resources; persons and hospitals interact to minimize several factors like number of fatalities, average waiting time, average ill-health, cost, etc. Likewise, the heuristics people employ to choose the hospital they should head to, based on prior knowledge about their size and location and real-time knowledge about current occupancies, can be seen as an extension of the Santa Fe bar problem [4]. Game theory also discusses different kinds of strategies that can effectively describe different personality, cultural and social traits governing panic behavior: some people imitate their neighbors, some are contrarian, some are rational, some are irrational, some employ a random strategy, etc.

Disaster planning is often based on assumptions derived from a conventional wisdom that is at variance with empirical field disaster research studies [3]. Our efforts to avert this error have resulted in a new system, called PLAN C (Planning with Large Agent-Networks against Catastrophes) [6, 7, 9, 12, 8], with well-identified, validated, simple rules with minimal number of parameters to avoid modeler bias and unnecessary complexity. The persons, hospitals, on-site responders, ambulances and disease prognosis follow deterministic rules with probabilistic parameters that can be modified by the user. A more detailed description of our system can be found in [7], where the Sarin gas exposure scenario is investigated in the constraints defined by Manhattan, New York, in [6], where the Brazilian food poisoning scenario is recreated and in [8] where various dynamics for different subpopulations and hospitals configurations are analyzed.

The system is implemented in Repast 3.1 [10], a popular and versatile JavaBased software toolkit that has been used to model such diverse concepts like intracellular processes and business strategies. We have also integrated ProActive $^{2}$ with RePast, in order to use the computational power of a cluster of computers to explore the parameter space of the system. Rather than focusing on the intricacies of the modeling problem, in this paper, we delve into the nature and sources of complexity in the dynamics of different kinds of catastrophes and various urban topologies.

\footnotetext{
${ }^{2}$ http://www-sop.inria.fr/oasis/proactive/
} 


\section{Experimental results}

In disaster management, it has been established that "Planning should take into consideration how people and organizations are likely to act, rather than expecting them to change their behavior to conform to the plan" [3]. ABM serves as a means of describing the behavior of medical facilities (controllable) and evaluating their performance in different disease scenarios for people with different personality and health profiles. Unless stated otherwise, results are carried out using the same values for the parameters as described in table 1 of the supplementary material ${ }^{3}$ and each plot is averaged on 10 independent runs.

Single event scenario As a first scenario, we consider a possible terrorist attack with a chemical warfare agent at Port Authority Bus Terminal in midtown Manhattan. In order to understand the complexity of the system dynamics, in Fig. 1, we monitor different statistics for the affected population. The left plot in Fig. 1 shows the evolution curves for the average waiting time of the affected population at the hospitals. The presence of three jumps is visible in the first 400 ticks of the curves, corresponding to the crowding effect of the flux of people at the three nearest hospitals to the site of the attack. Each climb phase is a consequence of the hospital state changing rapidly from "available" to "critical," with a resulting increase in the number of waiting non-critical persons. The flat phase that ensues is due to the state change from "critical" to "full", where all waiting persons are instructed to head to another hospital. It is interesting to
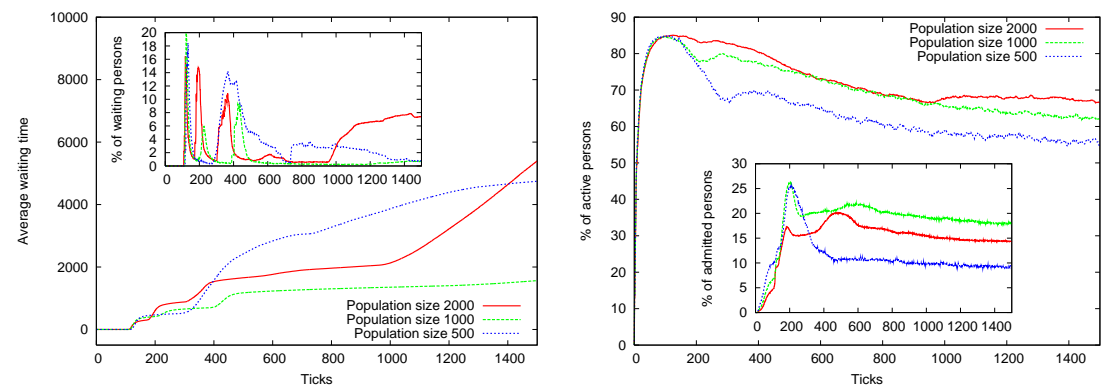

Figure 1: Left plot: evolution curves for the percentage of waiting persons at the hospitals and the average waiting time of the population with population size 500 , 1000 and 2000. Right plot: evolution curves for the percentage of active and admitted persons with population size 500, 1000 and 2000.

note how the population size of 500 persons seems to produce a more complex scenario as compared to a large size of 1000 , as evident in the higher waiting time at the hospitals. This unforeseen outcome can be explained by observing that after the nearest hospital becomes full, the remaining waiting population that heads to another hospital is unable to fill up the new one. The new hospital

${ }^{3}$ available at www.bioinformatics.nyu.edu/Projects/planc/appendix_iccs06.pdf 
remains in a critical state for more time causing an increased waiting time. This effect is visible in the inset plot on the left of Fig. 1, where the curve for the population size of 500 produces the highest percentage of waiting persons around 400 ticks. A similar behavior is produced by an affected population of 2000 individuals, but in this case the scenario unfolded after the three nearest hospitals became full.

The right plot of Fig. 1 shows the percentage of active and admitted persons. The term active denotes a person who has decided to head to a hospital. As expected, immediately after the attack, both the number of active and admitted persons quickly increases, but then different courses are produced by the different population sizes. Another unexpected behavior emerges in the right inset plot of Fig. 1: an affected population of 1000 individuals produces a higher percentage of admitted persons than that of 2000. A possible explanation can be found by observing that the resources of each hospital are the same for both population sizes, but the number of persons with lethal and severe injuries increases with the population size. These are persons who need more treatment producing a longer hospitalization time and higher demand of resources. At the same time, there are also many persons, some lightly and others severely injured, who are awaiting admission.

Multiple event scenario As a second scenario, we consider a possible terrorist attack involving multiple explosions - in particular, caused by three bombs located respectively in Union Square, Times Square and Central Park. The explosions are simulated to occur after 10, 120 and 300 minutes respectively. A population of 5000 persons is involved and initialized to random positions on the map at the beginning of the simulation. The left plot of Fig. 2 shows the
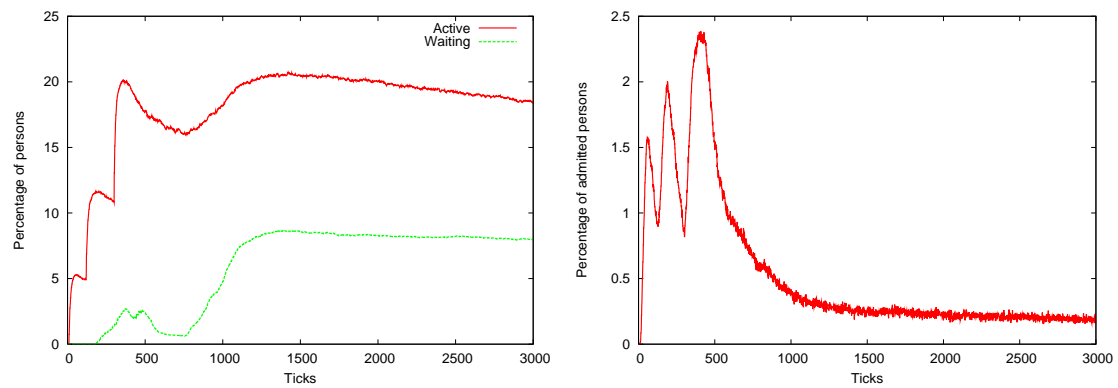

Figure 2: Left plot: evolution curves for active and waiting persons. Right plot: evolution curve for the percentage of admitted persons in the hospitals.

expected increase in the number of active persons after each of the three explosions. It is interesting to note the presence of an unpredictable fourth but less rapid increase after 1000 ticks. The waiting curve instead follows a completely different path because of the different spatial positions of the hospitals with respect to the sites of the explosions and their different resource levels. The right 
plot of Fig. 2 shows the curve for the percentage of admitted persons in the hospitals. As expected, after each explosion we have an increase in the number of admissions, but most of them are probably persons who do not need long-term hospitalization and hence, are discharged soon. However, the percentage of admitted persons never becomes zero; random fluctuations after the $700^{\text {th }}$ tick are visible due to the probabilistic personality factors (irrationality and compliance) of each person.

\subsection{Urban topologies and transportation}

The behavior of complex systems is strongly affected by the topology of the environment. This dependence has been observed in different domains (Biology, Social Science, Economy) and remains particularly true in the context of emergency response for a large urban environment. Location and distribution of the available resources can make the difference in the way a city responds to an attack. The topology of the streets and the transportation system affect how people make decisions as they travel to to their destination. Each city has different topological constraints and resource distributions, and a simulation framework needs to be flexible enough to be easily portable to different locations.
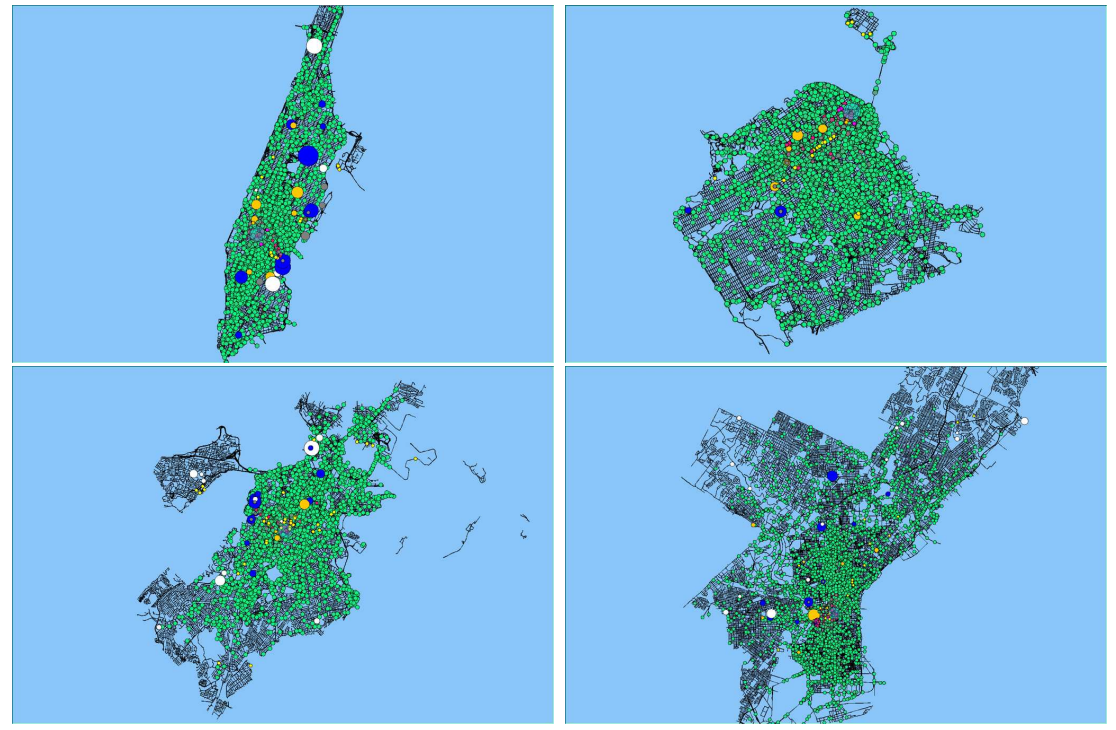

Figure 3: Warfare agent attack in 4 different U.S. cities (snapshots): left-top: New York City, NY; left-bottom: Boston, MA; right-top: San Francisco, CA; right-bottom: Philadelphia, PA.

Publicly available Geographic Information Systems (GIS) data about roads and transportation system of different cities was converted into a graph, where nodes are intersections and edges are streets. We have performed this conversion 
for 4 major cities in U.S.: Manhattan island (NYC), Boston (MA), San Francisco (CA) and Philadelphia in (PA). We have populated the model with hospital resources according to the data already included in the GIS source (if available) or according to publicly available web sites describing the hospital facilities. Agents are constrained to move only along the edges of the graph, with the effective speed at each time-step depending on the health level and probabilistic terms to simulate congestion effects. A simple variant of the $L R T A^{*}[5]$ algorithm for route computation is used to model a person's panic behavior.

We have compared the emergency dynamics of the same warfare agent attack with 5000 casualties in the downtown locations of each city. Fig. 3 shows a snapshot of the emergency scenario for each city analyzed, while Fig. 4 shows the dynamics of percentage of deceased and waiting persons for each city. Results show that San Francisco performs the worst among all the cities studied under almost identical attack scenario. This discrepancy is most likely due to the distribution of the hospitals, as in fact the majority of them are located far away from the downtown area. On the other hand, Philadelphia and Boston exhibit comparable performance in terms of fatalities, waiting and admissions to hospitals.
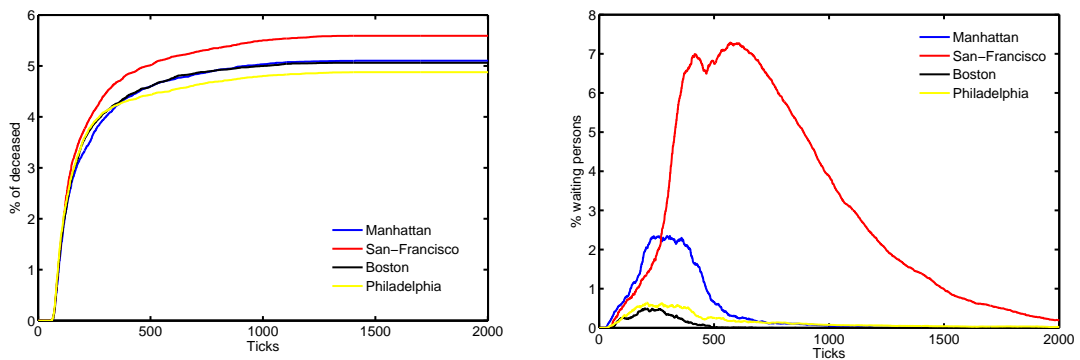

Figure 4: Left plot: percentage of deceased persons (by city). Right plot: percentage of people waiting for treatment at the hospitals (by city).

\section{$2.2 \quad$ ABM model-checking}

Unlike statistical analysis of metrics averaged over multiple agents and simulations, the model-checking approach focuses on individual agents' traces. Complex temporal properties may be described in Linear Temporal Logic (LTL) and then model-checked in a model-checker such as XSSYS[1]. The XSSYS system was originally developed for simulating and analyzing biochemical pathways. The agents' traces produced in output by the system can be read using XSSYS. To demonstrate the technique, we consider an intensive toxic agent exposure in downtown Manhattan and monitor a person and a hospital.

Fig. 5 shows two examples of queries that can be expressed in LTL for one of the hospital traces. For example, the query "EVENTUALLY(NUMBERADMITTED = 1)" returned TRUE, which means that there 
was a point in time when only one person was admitted inside hospital number 2. Clearly, very complex questions can be expressed in XSSYS and the trace analysis can help discover finer aspects about the underlying system dynamics.

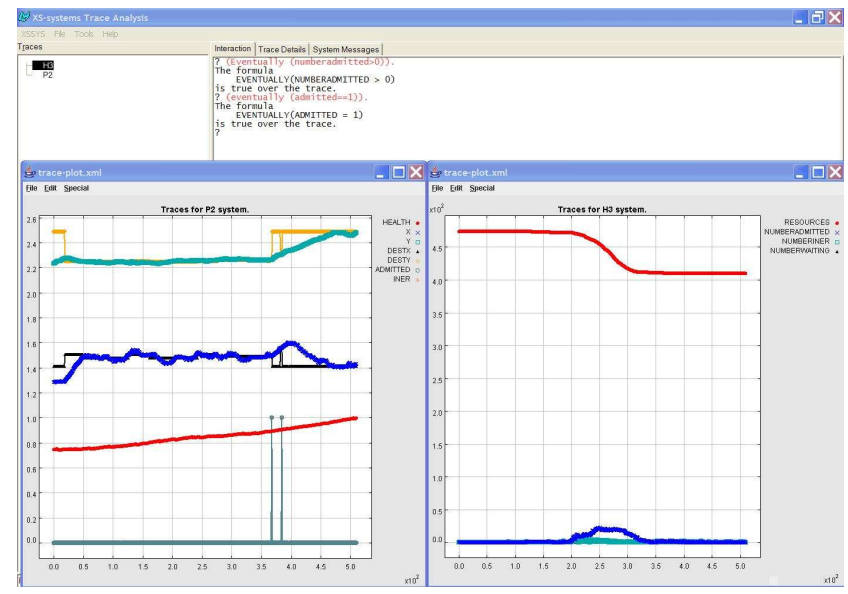

Figure 5: Temporal Logic Analysis in XSSYS. Left plot: Time-Trace of a Person. Right plot: Time-Trace of a Hospital.

\section{Multi-Objective Optimization for Planning}

Response plans involve different, often conflicting, criteria that must be satisfied and optimized in parallel - number of fatalities, average population health, time taken to succumb, waiting time at the hospital, life expectancy, economic cost, etc. In our framework, a response plan is expressed in terms of the system rules and parameters, producing a gargantuan strategy space that should be explored in order to find "optimal" plans. Moreover, the input parameters typically interact in a non-linear fashion. We have been exploring the use of multi-objective evolutionary algorithms (MOEAs) in order to devise plans that optimize multiple objective functions in terms of their Pareto frontier in the high-dimensional space defined by the system [9]. Over the last decade MOEAs have shown to have many of the properties needed to effectively tackle this challenging computational problems, such as their ability to: (i) generate multiple Pareto optimal solutions in a single run, (ii) handle a large search space, and (iii) provide robustness against the effects of noise. The PLAN C model produces, as part of its output, several of the relevant objectives/criteria involved in response planning in the form of statistical results of the global system behavior. In this context, a possible multi-objective formulation of the emergency response planning problem may be defined as follows: the selected input parameters of the model are the decision variables, the criteria for plan evaluation are the objectives, the parameter ranges are the variable bounds, and the mutual relations between 
the set of parameters are the constraints. In [9] we employed two well-known MOEAs, the Non-dominated Sorting Genetic Algorithm II (NSGA-II) and the Pareto Archived Evolution Strategy (PAES), and calibrated their performance for different pairs of objectives in the context of plan evaluation using PLAN C.

\section{Conclusions and future investigations}

The complex interactions between the affected population and the available resources of a response plan have remained poorly understood, are still beyond the analytical capability of traditional modeling tools, and have resisted any systematic investigation. In this research work we have shown that a deep analysis of the source of complexity generated by the simulation of different kind of urban emergency scenarios is effectively possible. This finer analysis has been accomplished using large-scale simulation with a novel Agent-Based Model simulation tool, PLAN C, addressing disasters in urban settings. Simple rules of behavior are seen to produce uncanny emergent dynamics with unpredictable interdependencies, which, with the help of the statistical analysis and optimization features of the system, can be inspected in order to refine existing plans and policies. Currently, we are extending the model in order to simulate not just immediate one-time events, e.g., attack with a chemical agent, bomb explosion, etc., but also long-lasting slowly-unfolding scenarios such as those resulting from an infectious disease, e.g., Smallpox.

Our efforts aim to demonstrate that the ABM paradigm, in conjunction with statistical analysis, multi-objective optimization, game theory and modelchecking of agent-traces, offers a novel way to understand, plan and control the unwieldy dynamics of a large-scale urban emergency response. The same empirical approach to mechanism design and selection in a complex repeated game will very likely find other applications: namely, (1) Social Networks, (2) Swarm robots, (3) Power systems design, (4) Synthetic and systems biology, etc. We are also exploring various research questions that these novel applications bring to the forefront.

\section{Acknowledgments}

We would like to acknowledge the funding support from Department of Homeland Security Grant \#2204-GT-TX-0001 and an NSF ITR grant \#CCR0325605. Ofer Gill currently at Bloomberg, Raoul-Sam Daruwala currently at Google and Fabian Menges currently at NYU Bioinformatics group also contributed to the implementation of PLAN C in RePast. We would like to thank our close collaborators Dr. Vijay Saraswat of IBM, Profs. Sanjoy Mitter and George Verghese of MIT and Peter Doerschuck of Cornell for their advice and criticisms. We would like to thank Silas Smith, Lewis Nelson, Dianne Rekow, Marc Triola, Liza Halcomb and Ian Portelli for their encouragement and input to the clinical aspect of the study design and development. 


\section{Bibliography}

[1] Antoniotti, M., F. Park, A. Policriti, N. Ugel, and B. Mishra, "Foundations of a query and simulation system for the modeling of biochemical and biological processes", The Pacific Symposium on Biocomputing: PSB (R. Altman, A. Dunker, L. Hunter, T. Jung, and T. Klein eds.), World Scientific (January 2003), 116-127.

[2] Batty, Michael, Cities and Complexity: Understanding Cities with Cellular Automata, Agent-Based Models, and Fractals, MIT Press (2005).

[3] DER HeIDE, E. Auf, "The importance of evidence-based disaster planning", Annals of Emergency Medicine 47, 1 (2006), 34-49.

[4] Greenwald, A., B. Mishra, and R. Parikh, "The santa fe bar problem revisited: Theoretical and practical implications", The Proceedings of the Summer Festival on Game Theory: International Conference, (1998).

[5] KorF, R.E., "Real-time heuristic search", Artificial Intelligence 42 (1990), 189-211.

[6] Mysore, Venkatesh, Ofer Gill, Raoul-Sam Daruwala, Marco AntoNiotti, Vijay SARAswat, and Bud Mishra, "Multi-agent modeling and analysis of the brazilian food-poisoning scenario", The Agent Conference, (2005).

[7] Mysore, Venkatesh, Giuseppe Narzisi, Lewis Nelson, Dianne Rekow, Marc Triola, Alan Shapiro, Clare Coleman, Ofer Gill, Raoul-Sam DARUWALA, and Bud Mishra, "Agent modeling of a sarin attack in manhattan", In Proceedings of the First International Workshop on Agent Technology for Disaster Management, ATDM (2006).

[8] Narzisi, Giuseppe, Joshua S. Mincer, Silas Smith, and Bud Mishra, "Resilience in the face of disaster: Accounting for varying disaster magnitudes, resource topologies, and (sub)population distributions in the plan c emergency planning tool", In Proceedings of the 3rd International Conference on Industrial Applications of Holonic and Multi-Agent Systems (HoloMAS 2007), vol. 4659, Springer LNAI (2007), 433-446.

[9] Narzisi, Giuseppe, Venkatesh Mysore, and Bud Mishra, "Multiobjective evolutionary optimization of agent based models: an application to emergency response planning", In Proceedings of the The IASTED International Conference on Computational Intelligence (CI 2006), ACTA press (2006), 224-230.

[10] North M.J., Collier N.T., and Vos J.R., "Experiences creating three implementations of the repast agent modeling toolkit", ACM Transactions on Modeling and Computer Simulation 16, 1 (2006), 1-25. 
[11] PNAS, Adaptive Agents, Intelligence, and Emergent Human Organization: Capturing Complexity through Agent-Based Modeling vol. 99(3), (May 2002).

[12] S.W., Smith, Nelson L.S., Rekow E.D., Narzisi G., Mincer J.S., Portelli I., Mishra B., and Goldfrank L.G., "A new approach to multi-hazard modeling and simulation", In Proceedings of the North American Congress of Clinical Toxicology (NACCT) 2007, vol. 45, Clinical Toxicology (2007). 